\title{
Novel method for rapid identification of micropapillary or solid components in early-stage lung adenocarcinoma
}

\author{
Ze-Rui Zhao, MD, ${ }^{\text {a,b }}$ Rainbow W. H. Lau, MBChB, FRCS, ${ }^{\mathrm{b}}$ Hao Long, MD, PhD, ${ }^{a}$ \\ Tony S. K. Mok, MD, FRCPC, ${ }^{\mathrm{c}}$ George G. Chen, PhD, ${ }^{\mathrm{b}}$ Malcolm J. Underwood, MBChB, FRCS, ${ }^{\mathrm{b}}$ and \\ Calvin S. H. Ng, MD, FRCS ${ }^{b}$
}

\begin{abstract}
Objective: Sublobar resection may be insufficient for early-stage lung adenocarcinoma with micropapillary or solid components because of the associated higher incidence of locoregional recurrence. This study sought to establish a novel method for rapidly identifying their presence to facilitate decision making for sublobar resection.
\end{abstract}

Methods: Antibody arrays of adhesion and apoptosis molecules were applied for adenocarcinomas with or without micropapillary/solid components to identify differentially expressed proteins. A semi-dry dot-blot system that visualizes the presence of target proteins was used to determine the presence of micropapillary or solid components in a prospective cohort of patients with clinical stage I who underwent operation. Sensitivity and specificity were calculated by comparing semi-dry dot-blot results with pathologic examinations.

Results: Insulin-like growth factor-binding protein 2 and P-cadherin were found more frequently in the micropapillary or solid positive group, and these were used as the target proteins in the semi-dry dot-blot system for detection of micropapillary or solid components. A total of 68 nodules with a mean size of $2.3 \pm 0.7 \mathrm{~cm}$, including $13(19.1 \%)$ with a micropapillary and $20(29.4 \%)$ with a solid pattern, were recruited. Micropapillary or solid $(+)$ lesions were more likely to have lymph node upstaging, greater diameter, and higher maximum standardized uptake value. The specificity and sensitivity for detecting the minor presence of micropapillary or solid component using the semi-dry dot-blot method were $94.4 \%$ (95\% confidence interval, 81.3-99.3) and $65.6 \%$ (95\% confidence interval, 46.8-81.4), respectively. The average test duration was $26.9 \pm 2.5$ minutes.

Conclusions: Detecting insulin-like growth factor-binding protein 2 and P-cadherin via the semi-dry dot-blot method could identify micropapillary or solid components in early-stage lung adenocarcinoma in a short processing time. (J Thorac Cardiovasc Surg 2018;156:2310-8)

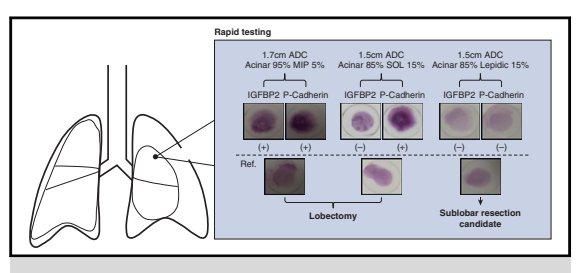

Chromogen density comparison detects the MIP/SOL pattern to guide resection type.

\section{Central Message}

Rapid detection of specific proteins may predict the presence of MIP/SOL component in early-stage lung ADC.

\section{Perspective}

Lung ADCs with MIP or SOL patterns have a higher incidence of locoregional recurrence after sublobar resection. A fast and reliable method for identifying these components is not well characterized. Rapid detection of IGFBP-2 and P-cadherin via the SBD method achieves high specificity and moderate sensitivity in predicting their presence.
The role of intentional sublobar resection in the treatment of early-stage lung cancer is still a matter of debate, ${ }^{1}$ with randomized trials (JCOG0802 and CALGB-140503) designed to address this issue completing in the coming 5 years. ${ }^{2,3}$

From the a State Key Laboratory of Oncology in Southern China, Collaborative Innovation Centre for Cancer Medicine, and Department of Thoracic Surgery, Sun YatSen University Cancer Centre, Guangzhou, China; 'bivision of Cardiothoracic Surgery, Department of Surgery, The Chinese University of Hong Kong, Prince of Wales Hospital, Hong Kong SAR, China; and ${ }^{\mathrm{c} D e p a r t m e n t ~ o f ~ C l i n i c a l ~}$ Oncology, The Chinese University of Hong Kong, Prince of Wales Hospital, Hong Kong SAR, China.

This work was supported by Research Grants Council General Research Fund [14117715], Chinese University of Hong Kong Direct Grant [2015.1.087], and Foundation for Science and Technology Research Project of Guangdong [2014B020212014].
Most of the reported series comparing sublobar resection with lobectomy, including these 2 ongoing trials, ${ }^{2,3}$ included lesions no more than $2 \mathrm{~cm}$ in diameter. However, accumulating evidence has shown that different subtypes 


\section{Abbreviations and Acronyms \\ $\mathrm{ADC}=$ adenocarcinoma \\ CI $=$ confidence interval \\ $\mathrm{CT}=$ computed tomography \\ IGFBP2 = insulin-like growth factor-binding protein 2 \\ MIP = micropapillary \\ PBS = phosphate-buffered saline \\ SDB $=$ semi-dry dot-blot \\ $\mathrm{SOL}=$ solid}

SUVmax $=$ maximum standardized uptake value

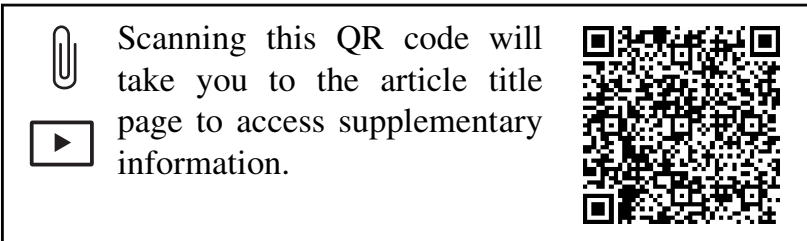

of invasive adenocarcinoma (ADC) significantly affected tumor recurrence and long-term survival. ${ }^{4,5}$ Of particular interest, ADCs with even minor micropapillary (MIP) or solid (SOL) components had shorter disease-free survival, as well as higher rates of locoregional recurrence, after sublobar resection, and therefore a more radical resection could be warranted in these patients. ${ }^{6}$

Traditional methods, including preoperative tumor centesis, ${ }^{7}$ radiologic examination, ${ }^{8}$ and examination of intraoperative frozen sections have limitations for detecting MIP/SOL patterns within the tumor to decide on the extent of resection. ${ }^{9,10}$ Other means that provide a rapid detection of these ADC subtype patterns with unfavorable outcome are required. The present study was performed to establish a novel kit based on the dot-blot technique for the rapid detection of MIP/SOL components in earlystage lung ADC. Theoretically, the novel diagnostic kit would be simple to handle and could alleviate the workload of onsite pathologists to distinguish ADC subtypes. A prospective cohort was also recruited to evaluate its diagnostic performance.

\section{MATERIALS AND METHODS}

\section{Patients and Morphologic Assessment}

Specimens for array and Western blotting analyses were obtained from the tumor bank of Sun Yat-Sen University Cancer Center and Prince of Wales Hospital. These cases were stage I to IIIa lung ADC that underwent surgical resection. Subsequently, a prospective cohort consisting of patients with clinical stage I ADC with a tumor diameter $2 \mathrm{~cm}$ or less in radiologic examination with subsolid appearance and underwent lung surgery from January 2016 to March 2017 were enrolled for diagnostic validation. Resected specimens were reviewed according to the 2015 World Health Organization classification, and each component (lepidic, papillary, acinar, MIP, and SOL) was recorded in 5\% increments. ${ }^{11,12}$ Visceral pleural invasion was assessed in all cases, and elastic stain was used in cases with indeterminate results on hematoxylin-eosin staining. ${ }^{13}$ Patients provided written informed consent, and the study protocol was approved by the local ethical review boards (approval Institutional Review Board number 2015.1.087).

\section{Identifying Proteomic Differences Among Adenocarcinoma Subtypes}

Previous reports mentioned the potential alteration of adhesion and apoptosis molecules in MIP/SOL subtypes in comparison with other components. ${ }^{14-16}$ Relevant antibody arrays (Human Adhesion Molecule Array G1 and Human Apoptosis Array G1 screening 17 and 44 proteins, respectively, from the RayBio Human Protein Array G Series; RayBiotech, Norcross, Ga) (Tables E1 and E2) were applied for 8 ADCs with ( $\geq 5 \%$ by histology, test) and 8 without $(<5 \%$ by histology, control) MIP/SOL components to identify differentially expressed proteins. Tumor specimens were lysed and dialyzed with phosphate-buffered saline (PBS) and added to the array membranes. Proteins that were captured by the membrane-bound antibodies were detected by incubation with secondary biotinylated antibody and analysis by fluorescence detection. Normalization was performed using the signals of internal controls on each array chip. Significance was determined by $t$ test with a fold-change index in signal of at least 1.5. Candidate protein selection was based on the rationale that it should have been reported previously as being differentially expressed at the mRNA or protein level in human cancer. These proteins were further validated by Western blotting analysis in an independent cohort ( 34 cases in test vs 14 cases in control).

\section{Semi-Dry Dot-Blot System for Detecting Target Proteins}

Briefly, after removal of the tumor intraoperatively, a small block of tumor was cut into 3 parallel sections every 3 to $4 \mathrm{~mm}$ along the maximum diameter. Divided tumors were then washed for 30 seconds with $2 \mathrm{~mL}$ of PBS; the lavage fluid was centrifuged to collect suspended cells, which were then lysed (RIPA lysis buffer; Millipore, Billerica, Mass). The dot-blot membrane (modified nylon membrane, Immunodyne ABC; Pall, Ann Arbor, Mich) was soaked in wash buffer ( $0.05 \%$ Tween 20 in PBS). Antibodies selected in the previous step were used as the primary antibodies (monoclonal antibodies to P-cadherin and insulinlike growth factor-binding protein 2 [IGFBP2]; Abcam, Cambridge, UK), and peroxidase-coupled immunoglobulin G (ImmPRESS antimouse/rabbit immunoglobulin G; Vector Laboratories, Burlingame, Calif) was used as the secondary antibody. Both primary and secondary antibodies were placed onto 1 circle for the actual examination, and primary antibody alone was placed onto the other circle as a reference. The membrane was incubated with the 2 antibodies for 3 minutes at room temperature. The results were visualized using a Vector VIP substrate kit (Vector Laboratories), which produces a purple chromogen in the presence of peroxidase activity. A positive result was denoted by a denser appearance of chromogen in the circle of the actual examination than in the reference circle. In contrast, a negative result was shown by an equal density of chromogen in both circles (Figure 1). ${ }^{17}$ See highlights of the study in the Video 1.

\section{Statistical Analysis}

Continuous data are expressed as means and were compared using the Student $t$ test. Categoric data were compared using the chi-square test or Fisher exact test, as appropriate. The accuracy of the semi-dry dot-blot (SDB) technique for detecting MIP or SOL components of lung ADC intraoperatively was determined on the basis of the gold standard, that is, pathologic examination. Sensitivity, specificity, and accuracy were determined by comparing the results obtained with the SDB kit with those of the pathologic examinations of the relevant tumor specimens with $95 \%$ confidence 


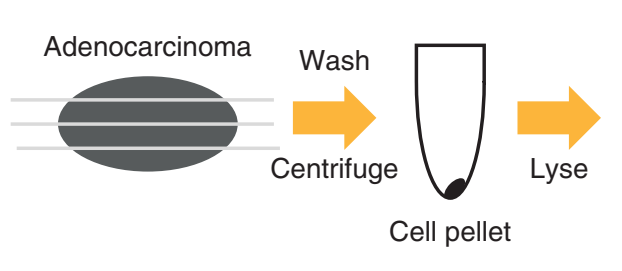

Colorimetric detection on membrane
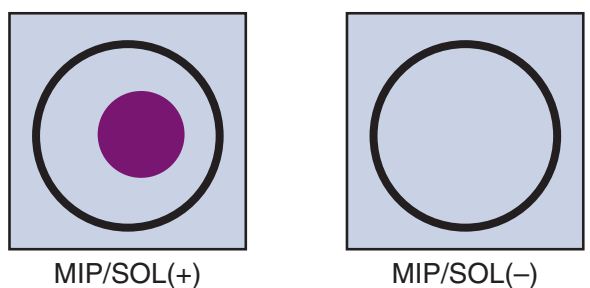

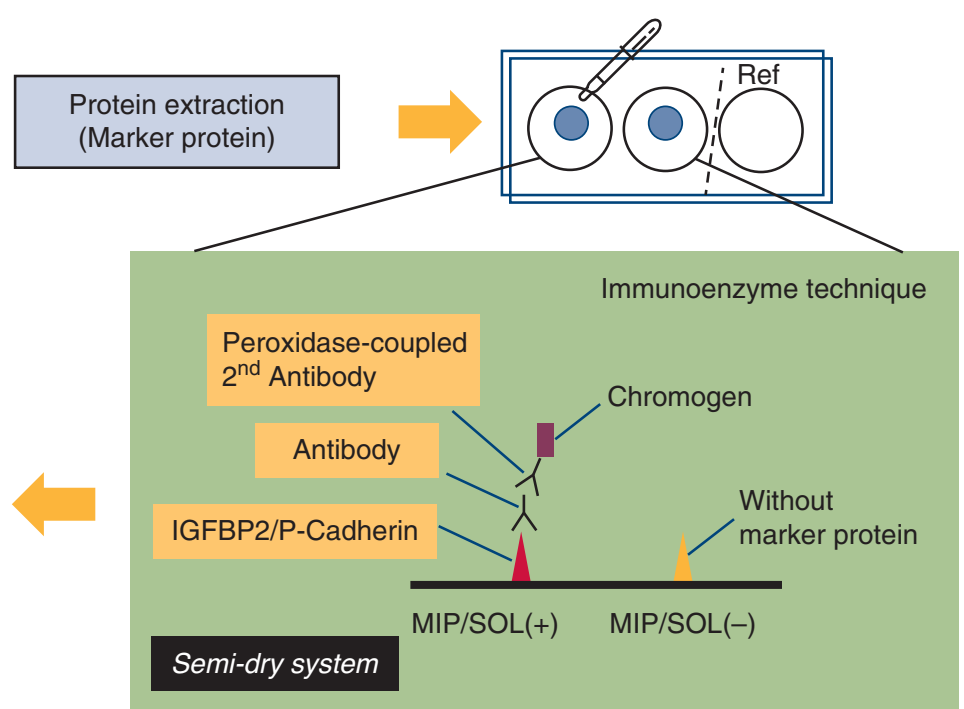

FIGURE 1. Illustration of the SDB system. ADC tissue is cut with series parts and washed with PBS. After centrifugation, the tumor cells are lysed for protein extraction. The samples are then added to the dot-blot membrane that was incubated with anti-IGFBP2 and anti-P-cadherin antibody under a semidry condition. The result is read by colorimetric comparison through a chromogen. IGFBP2, Insulin-like growth factor-binding proteins $2 ;$; $I P$, micropapillary; SOL, solid.

intervals (CIs). All analyses were conducted using PASW statistical software (ver. 20.0; SPSS Inc, Chicago, Ill).

\section{RESULTS}

\section{Differently Expressed Proteins Between Positive and Negative Micropapillary/Solid}

The antibody arrays were used to profile 4 ADCs with MIP components (median, 26.3\%; range, 5-70), as well as 4 other ADCs with SOL components (median, 27.5\%; range, 10-40) and $8 \mathrm{MIP} / \mathrm{SOL}$ negative cases. Through these analyses, 4 differentially expressed proteins were identified (Figure 2, $A$ and $B$ ): soluble tumor necrosis factor receptor 1 , heat shock protein 27 , IGFBP2 on the apoptosis array (fold-changes: $1.62,1.86$, and $3.14 ; t$ test $P$ values: .05 , .02 , and $<.01$, respectively), and P-cadherin on the adhesion array (fold-change: $2.46 ; t$ test $P$ value: .03; Table E3).

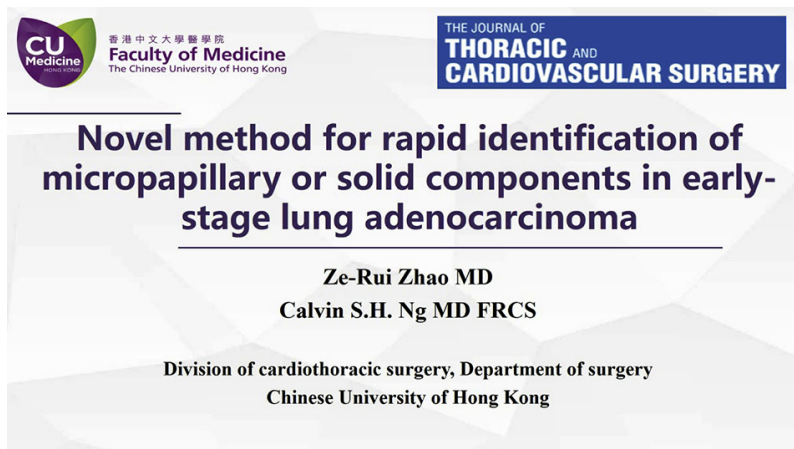

VIDEO 1. Summary of the current study. Video available at: https://www. jtcvs.org/article/S0022-5223(18)32045-2/fulltext.
Further validation showed that the relative expression levels of IGFBP2 $(P<.05)$ and P-cadherin $(P=.02)$ measured by the array and Western blotting (MIP component: $3.14 \% \pm 8.36 \%$, SOL component: $7.89 \% \pm 18.21 \%$ ) were similar (Figure 2, C). On the contrary, there was no difference regarding the relative expression level of heat shock protein $27(P=.07)$ or soluble tumor necrosis factor receptor $1(P=.40)$. Therefore, IGFBP2 and P-cadherin were selected as MIP/SOL-associated markers.

\section{Use of Semi-Dry Dot-Blot Kit for Detecting Micropapillary/Solid Components}

Colorimetric differences in either 1 of the spotted circles were used to define SDB positivity (Table E4). A positive result indicated the presence of IGFBP2/P-cadherin and indirectly supported the potential presence of MIP/SOL components within a tumor (Figure 2, D). Of note, the test successfully identified 1 of the 2 synchronous lesions in a patient who had SOL components (Figure 3 ).

\section{Prospective Cohort for the Semi-Dry Dot-Blot Test and Patient Characteristics}

A total of 68 ADCs with a mean size of $2.3 \pm 0.7 \mathrm{~cm}$ were enrolled to evaluate the SDB test, including $13(19.1 \%)$ with a MIP and $20(29.4 \%)$ with a SOL pattern (1 tumor had both MIP and SOL component). All cases underwent lobectomy except for 1 patient who received bilobectomy for the removal of his synchronous tumors. Most of the cases $(60 / 68,88.2 \%)$ were non-MIP/SOL predominant cancers, and only 1 case $(1.5 \%)$ had both MIP and SOL components within the same tumor. Lesions with MIP/ 


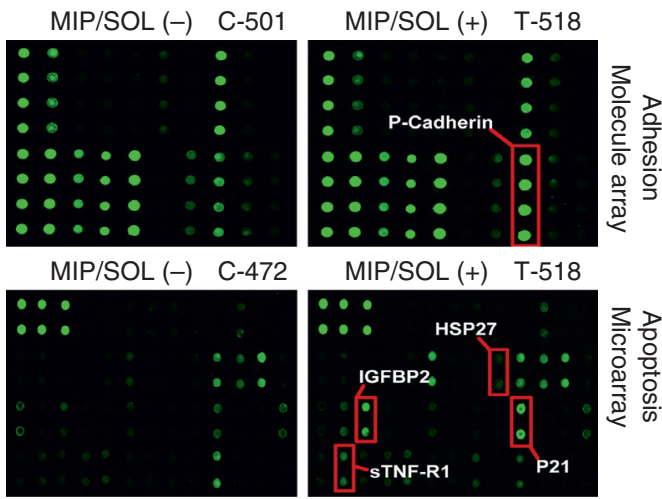

A
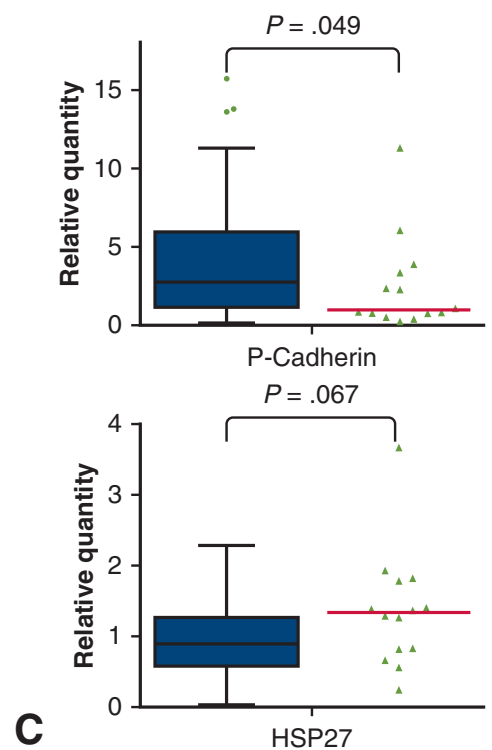
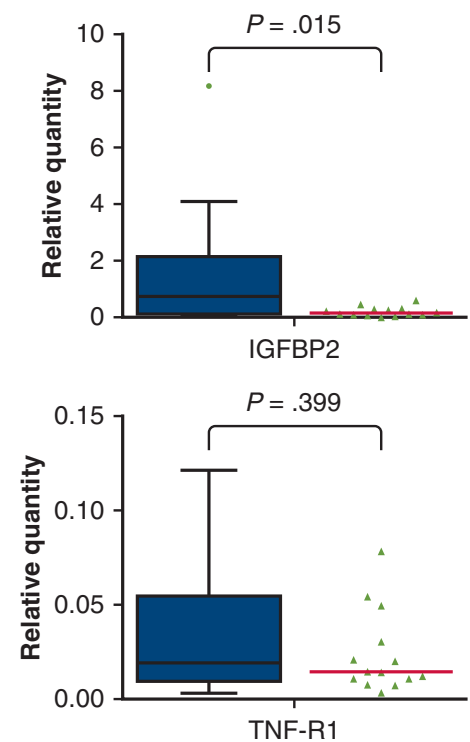

TNF-R1

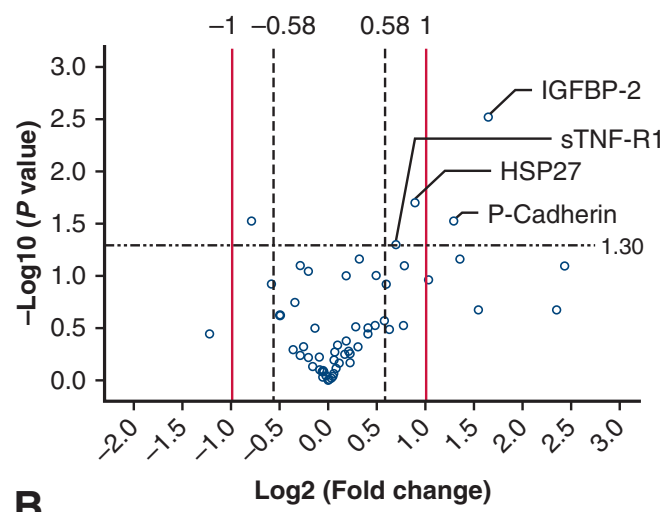

MIP/SOL positive $(n=34)$

$\triangle \mathrm{MIP} / \mathrm{SOL}$ negative $(\mathrm{n}=14)$

Positive control Negative control

$1.7 \mathrm{~cm} \mathrm{ADC}$ Acinar 95\% MIP 5\% Acinar $85 \%$ SOL $15 \%$ Acinar $85 \%$ Lepidic $15 \%$

$1.5 \mathrm{~cm}$ ADC MIP/SOL (+) case from MIP/SOL (-) case from

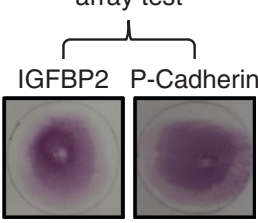
array test

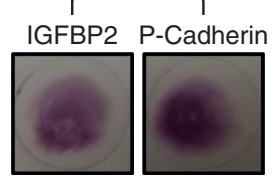

$(+)$

$(+)$

$(-)$

$(+)$

$(-)$

$(-)$
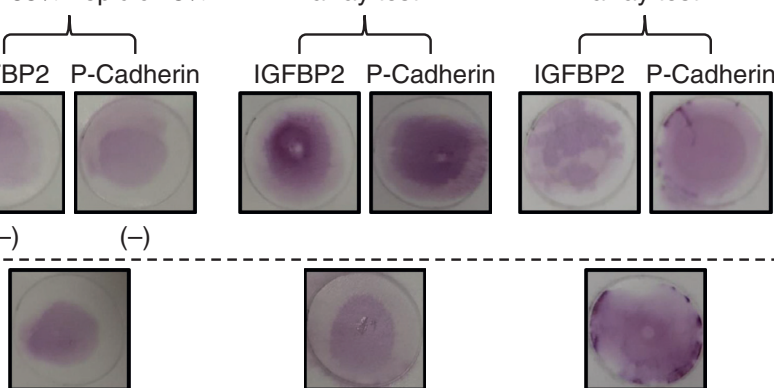

D
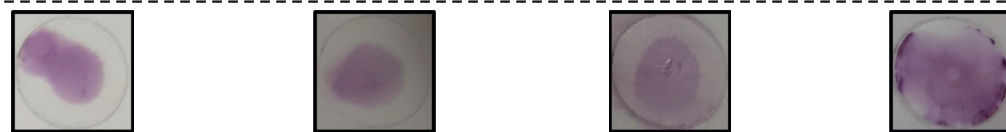

FIGURE 2. A, Antibody arrays of the adhesion and apoptosis pathway showed different expressions between MIP/SOL positive ( $\geq 5 \%$, test group [T]) and MIP/SOL negative ( $<5 \%$, control group [C]). B, Volcano plots summarized 4 alterations with fold changes greater than 1.5 or 2.0 located at the right side of 0.58 or 1 in horizontal axis, as well as $P$ values less than .05 to be above $1.30 \mathrm{in}$ vertical axis by analyzing the T/C results from the antibody array. Note that each circle represents a protein to be screened in the arrays. C, Western blot validated P-cadherin and IGFBP2 to be the target proteins between T and C. The upper and lower borders of the box represent the upper and lower quartiles in group T. The middle horizontal line represents the median. The upper and lower whiskers represent the maximum and minimum values of nonoutliers (75th percentile plus $1.5 *$ interquartile range and 25 th percentile minus $1.5 *$ interquartile range). Extra dots represent outliers. The number of subjects in group C was less than 15; thus, each of the data points is plotted with a line for the median value. D, Chromogen density comparison among P-cadherin, IGFBP2, and reference in the SBD test gave a rapid impression of protein expression, thus indicating potential existence of MIP/SOL components. MIP, Micropapillary; SOL, solid; HSP, heat shock protein; IGFBP2, insulin-like growth factorbinding proteins 2 ; $s T N F-R 1$, soluble tumor necrosis factor receptor $1 ; A D C$, adenocarcinoma. 

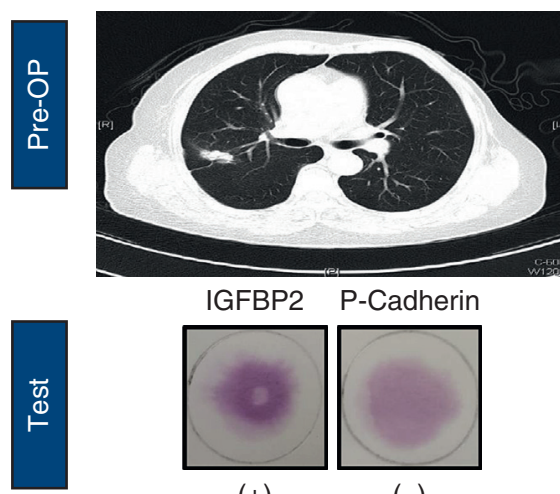

$(+)$

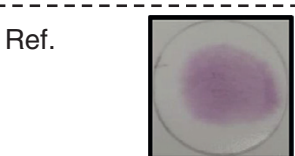

Right upper lobe lesion SOL $70 \%$ Acinar $30 \%$

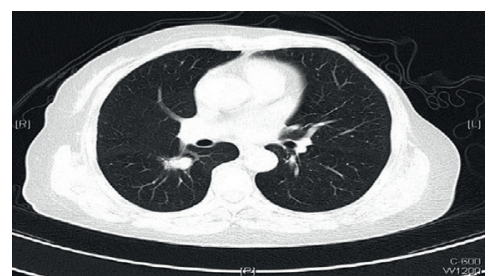

IGFBP2 P-Cadherin

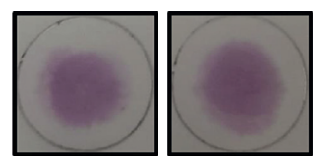

$(-)$
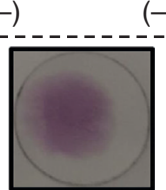

Right lower lobe lesion Acinar $95 \%$ Papillary $5 \%$

FIGURE 3. The diagnostic kit successfully identified 1 of the synchronic pulmonary lesions in a single patient who had $70 \%$ of solid growth component. $I G F B P 2$, Insulin-like growth factor-binding proteins 2; SOL, solid.

SOL components were more likely to have lymph node metastases, greater tumor diameter, and a higher maximum standardized uptake value (SUVmax) on preoperative positron emission tomography/computed tomography (CT) (Table 1). Eleven lesions (34.4\%) with MIP/SOL components had an SUVmax greater than 7, compared with 6 lesions $(16.7 \%)$ in the MIP/SOL-negative group $(P=.16)$. Thirteen cases $(19.1 \%)$ had nodal upstaging to N1 station only, with the majority of them involved with the hilar region $(11 / 13,84.6 \%)$. Two MIP/SOL-negative ADCs had hilar lymph node metastasis. In addition, N2 station involvement $(\mathrm{N}=2,2.9 \%)$ was found exclusively in

TABLE 1. Tumor characteristics of the prospective cohort

\begin{tabular}{|c|c|c|c|c|}
\hline & Total $(N=68)$ & $\begin{array}{c}\text { Without MIP/SOL } \\
\text { component }(\mathbf{N}=36)\end{array}$ & $\begin{array}{c}\text { With MIP/SOL } \\
\text { component }(\mathbf{N}=32)\end{array}$ & $\boldsymbol{P}$ \\
\hline Age, $y$ & $63.49 \pm 8.4$ & $63.36 \pm 6.4$ & $63.63 \pm 9.98$ & .90 \\
\hline Female $(\mathrm{N}, \%)$ & $50,73.5 \%$ & $26,72.2 \%$ & $24,75.0 \%$ & .80 \\
\hline $\begin{array}{l}\text { Predominant subtype } \\
\text { Lepidic } \\
\text { Acinar } \\
\text { Papillary } \\
\text { MIP } \\
\text { SOL }\end{array}$ & $\begin{array}{r}6,8.8 \% \\
44,64.7 \% \\
14,20.6 \% \\
1,1.5 \% \\
3,4.4 \%\end{array}$ & $\begin{array}{r}4,11.1 \% \\
24,66.7 \% \\
8,22.2 \% \\
\text { NA } \\
\text { NA }\end{array}$ & $\begin{array}{r}2,6.3 \% \\
20,62.5 \% \\
6,18.8 \% \\
1,3.1 \% \\
3,9.4 \%\end{array}$ & .30 \\
\hline Lymph node metastasis ( $\mathrm{N}, \%)$ & $15,22.1 \%$ & $2,5.6 \%$ & $13,40.6 \%$ & $<.01$ \\
\hline Cribriform component $(\mathrm{N}, \%)$ & $5,7.4 \%$ & $2,5.6 \%$ & $3,9.4 \%$ & .66 \\
\hline Lymphovascular invasion $(\mathrm{N}, \%)$ & $9,13.2 \%$ & $4,11.1 \%$ & $5,15.6 \%$ & .73 \\
\hline Visceral pleural invasion (N, \%) & $10,14.7 \%$ & $3,8.3 \%$ & $7,21.9 \%$ & .17 \\
\hline Tumor size $(\mathrm{cm})$ & $2.30 \pm 0.73$ & $2.13 \pm 0.61$ & $2.49 \pm 0.82$ & .045 \\
\hline SUVmax & $5.43 \pm 3.80$ & $4.19 \pm 3.15$ & $6.84 \pm 4.02$ & $<.01$ \\
\hline GGO component on $\mathrm{CT}(\%)$ & $16.91 \pm 18.47$ & $19.44 \pm 17.84$ & $14.06 \pm 19.03$ & .23 \\
\hline MIP component $(\%)^{*}$ & - & NA & $2.79 \pm 8.74$ & NA \\
\hline SOL component $(\%) \dagger$ & - & NA & $7.43 \pm 17.61$ & NA \\
\hline
\end{tabular}

Bold values indicate statistical significance. $M I P$, micropapillary; $S O L$, solid; $N A$, not available; $S U V$, standardized uptake value; $G G O$, ground-glass opacity; $C T$, Computed tomography. $* \mathrm{~N}=13 . \dagger \mathrm{N}=20$. 
TABLE 2. Comparison of the semi-dry dot-blot method and pathologic examination in the detection of micropapillary/solid components in early-stage lung adenocarcinoma

\begin{tabular}{lccc}
\hline & \multicolumn{2}{c}{ Pathologic assessment } & \\
\cline { 2 - 3 } & MIP/SOL positive & MIP/SOL negative & Sum \\
\hline SDB test & & 2 & \\
Positive & 21 & 34 & 23 \\
Negative & 11 & 36 & 45 \\
Sum & 32 & 68 \\
\hline
\end{tabular}

$M I P$, Micropapillary; $S O L$, solid; $S D B$, semi-dry dot-blot test.

the MIP/SOL-positive group. In contrast, there were no differences in age, gender, cribriform component, lymphovascular invasion, visceral pleural invasion, or ground-glass opacity percentage on CT between tumors with versus without MIP/SOL patterns.

\section{Performance of the Test}

The average test duration was $26.9 \pm 2.5$ minutes for the 68 lesions examined by the SDB test, with an accuracy of $80.9 \%$ (95\% CI, 69.5-89.4) (Table 2). A total of 34 of the 36 pathologically MIP/SOL-negative lesions were negative on the SDB test (specificity, $94.4 \%$; 95\% CI, 81.3-99.3). A total of 21 of the 32 pathologically MIP/SOL-positive lesions were detected by the SDB method (sensitivity, $65.6 \%$; 95\% CI, 46.8-81.4). The positive and negative predictive values were $91.3 \%$ (95\% CI, 72.7-97.6) and 75.6\% (95\% CI, 65.6-83.4), respectively. The sensitivity of the test to identify MIP and SOL pattern separately was $69.2 \%$ (95\% CI, 38.6-90.9\%) and 60.0\% (95\% CI, 36.1-80.9), respectively (Table E5).

In addition, there was no significant difference in tumor diameter between cases with incorrectly $(2.62 \pm 0.93 \mathrm{~cm})$ and correctly diagnosed SDB cases $(2.22 \pm 0.67 \mathrm{~cm}$, $P=.08)$. In the 11 false-negative cases, 5 of them $(45.4 \%)$ had N1 station lymph node metastasis, which was higher than in the true-positive cases $(6 / 21,28.6 \%)$. However, this did not reach statistical significance $(P=.44)$. Additionally, $\mathrm{N} 2$ involvement $(\mathrm{N}=2)$ was only found in tumors with true-positive results.

\section{DISCUSSION}

An increasing number of lesions treated by pulmonary resection in our practice were part-solid lesions on CT scan. Such lesions can be invasive ADCs even if their diameter is small. According to the new ADC classification, however, more than $80 \%$ of invasive ADCs may have a mixed subtype growth pattern. ${ }^{5,18}$ More important, nonpredominant growth patterns, that is, MIP/ SOL components accounting for $5 \%$ or greater of the entire tumor, are frequently associated with lymphovascular invasion, visceral pleural involvement, and mediastinal lymph nodes metastasis and thus negatively affect the clinical outcome. Although MIP/ SOL positive cases had a higher percentage of patients with lymphovascular and visceral pleural invasion, as well as lower ground-glass opacity components, no significant difference was noticed in this study, which may derive from the limited number of patients enrolled. Our previous study indicated that early-stage acinar/papillarypredominant ADCs with minor MIP/SOL components had similar disease-free and overall survival to MIP/ SOL-predominant cases, which had the poorest survival among all ADC growth subtypes. ${ }^{5}$ Nitadori and colleagues ${ }^{19}$ also reported that the presence of MIP was associated with increased risk of locoregional recurrence in stage I ADCs no more than $2 \mathrm{~cm}$ in diameter treated by limited resection than those without (5-year cumulative incidence of recurrence: $34.2 \%$ vs $12.4 \%$ ). The MIP component did not affect the recurrence hazards in patients who received lobectomy. Therefore, identification of the presence of a minor MIP/SOL pattern in earlystage lung ADCs could help surgeons make appropriate choices with respect to the extent of resection in patients for whom intentional sublobar resection is planned. ${ }^{6,20}$ For instance, patients with synchronous lung ADCs could choose lobectomy for the MIP/SOL $(+)$ lesion and segmentectomy/wedge resection for the MIP/SOL (-) lesion to preserve pulmonary function (Figure 3).

The presence of MIP/SOL components in stage I ADCs ranges from $9.5 \%$ to $46.8 \%$ in the literature and was $47.1 \%$ in the present study. ${ }^{6,9}$ Several methods to predict their presence have been reported to date. By investigating multiple clinical factors, Cha and colleagues $^{8}$ suggested that MIP/SOL was more common in lesions greater than c-stage I, and with a size $2.5 \mathrm{~cm}$ or greater, solid appearance on CT, and SUVmax 7 or greater. However, tumors with such characteristics may not be eligible for intentional sublobar resection. Consistent with the published data, SUVmax was higher in the MIP/SOL $(+)$ group in this study. But only $34.4 \%$ of these lesions had an SUVmax greater than 7, suggesting that SUV alone may not be sufficient for prediction. Huang and colleagues also reported unsatisfactory accuracy of preoperative biopsy for identifying the presence of ADC subtypes in 128 patients undergoing pulmonary resection. The sensitivity of detecting MIP/SOL components was as low as $16.5 \%$, and the biopsy failed to detect MIP/SOL components in $86 \%$ of cases, even with MIP/SOL components accounting for more than $40 \%$ of the entire tumor.

Considering the heterogeneity of a tumor, frozen-section examination during surgery may provide better information than preoperative methods. Yeh and colleagues ${ }^{9}$ and Trejo Bittar and colleagues ${ }^{10}$ compared the concordance rates of 
TABLE 3. Summary of the reported publications for the detection of nonpredominant micropapillary/solid components in early-stage lung adenocarcinoma

\begin{tabular}{|c|c|c|c|c|c|c|}
\hline Authors & Methodology & Stage & $\mathbf{N}$ & $\begin{array}{c}\text { Concordance rate } \% \\
(95 \% \text { CI })\end{array}$ & $\begin{array}{l}\text { Sensitivity \% } \\
(95 \% \text { CI })\end{array}$ & $\begin{array}{c}\text { Specificity \% } \\
(95 \% \text { CI })\end{array}$ \\
\hline $\begin{array}{l}\text { Huang and } \\
\quad \text { colleagues }^{7} 2017\end{array}$ & Preoperative biopsy & cStage I-IV & 128 & $47.7(39.2-56.3)$ & $16.5(9.1-26.5)$ & $98.0(89.2-99.9)$ \\
\hline \multirow[t]{2}{*}{$\begin{array}{l}\text { Cha and colleagues } \\
2014\end{array}$} & $\begin{array}{l}\text { Multi preoperative } \\
\text { factors }\end{array}$ & cStage I-IIIa & 511 & NA & NA & NA \\
\hline & \multicolumn{6}{|c|}{ Note: Greater than stage I, tumor size $\geq 2.5 \mathrm{~cm}$, solid mass, and SUVmax $\geq 7$ predicted MIP/SOL presence. } \\
\hline $\begin{array}{l}\text { Trejo Bittar and } \\
\text { colleagues }^{10} 2015\end{array}$ & Frozen section & pStage I & 112 & NA & $\begin{array}{l}\text { MIP: } 13.3(0.3-26.4) \\
\text { SOL: } 50(12.6-87.4)\end{array}$ & $\begin{array}{l}\text { MIP: } 98.1(96-100) \\
\text { SOL: } 95.2(94.1-96.3)\end{array}$ \\
\hline $\begin{array}{l}\text { Yeh and colleagues } \\
2015\end{array}$ & Frozen section & pStage I & 361 & $\begin{array}{l}\text { MIP: } 67(62-72) \\
\text { SOL: } 84(80-88)\end{array}$ & $\begin{array}{l}\text { MIP: } 37(30-45) \\
\text { SOL: } 69(61-76)\end{array}$ & $\begin{array}{l}\text { MIP: } 94 \text { (89-97) } \\
\text { SOL: } 96 \text { (92-98) }\end{array}$ \\
\hline Current study & SDB kit & cStage I & 68 & $80.9(69.5-89.4)$ & $65.6(46.8-81.4)$ & $94.4(81.3-99.3)$ \\
\hline
\end{tabular}

frozen sections and permanent pathologic examination for the detection of MIP/SOL in 473 p-stage I ADCs. ${ }^{9,10}$ Their results indicated that the specificity of frozen sections was encouraging (range, 94\%-98\%), whereas the sensitivity was relatively low (range, $13.3 \%-37 \%$ for MIP and $50 \%-69 \%$ for SOL). Furthermore, only moderate interobserver agreement was found, which indicated that the discrepancy between the frozen sections and final diagnosis could be due to sampling error and quality. The present study indicated similar sensitivity and specificity compared with the frozen sections, thus highlighting the importance of sampling the largest representative part of a tumor whenever possible (Table 3). Likewise, the interpretation of a negative SDB test result should be cautious considering that lobectomy is still a standard operation for patients with operable lung cancer. Only some of the patients would safely benefit from undergoing a sublobar procedure according to the SDB result because the current test yields a moderate sensitivity. Nevertheless, the SDB method may have certain advantages over frozen section. On the one hand, the processing and reading of the test are simple; thus, a staff who received short-term training can apply the technique easily that may reduce interobserver bias. On the other hand, having an onsite pathologist with senior experience of distinguishing ADC subtypes from frozen section may not always be available.

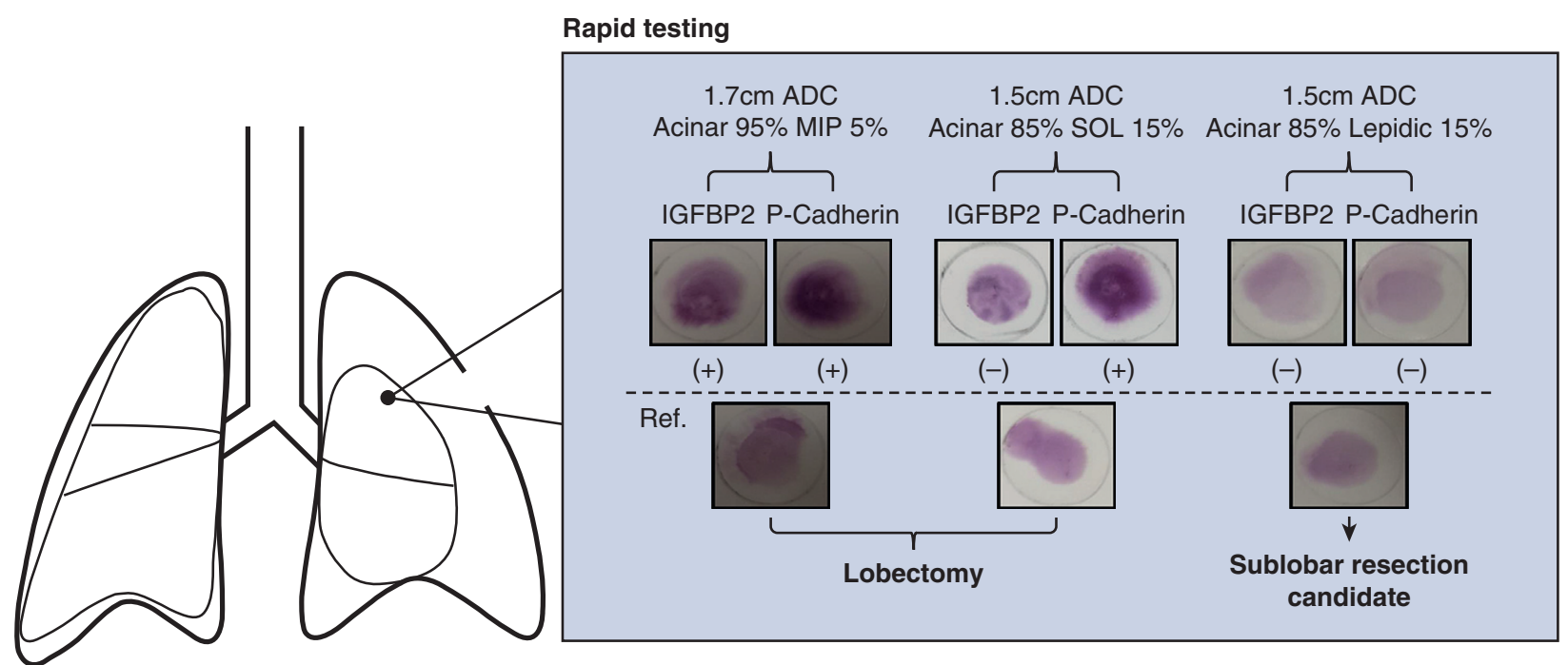

FIGURE 4. Chromogen density comparison detects MIP/SOL pattern to guide resection type for early-stage lung cancer: Patients with the presence of these 2 growth components are suggested to undergo lobectomy, whereas those without can choose sublobar resection. ADC, Adenocarcinoma; $M I P$, micropapillary; IGFBP2, insulin-like growth factor-binding proteins 2. 
The SDB method, first introduced by Hirakawa and colleagues ${ }^{17}$ in 2010, can visualize the presence of lymph node metastasis by detecting anti-pancytokeratin as an indicator of cancer cells. The short experiment time ( $\sim 30-40$ minutes) and high sensitivity, capable of detecting as little as $0.01 \mathrm{mg} / \mathrm{mL}$ of protein extracted from tissue, makes it possible for intraoperative diagnosis, and this method has been applied for both lung and breast cancers. ${ }^{21,22}$ By studying the antibody arrays of MIP/SOL $(+)$ and MIP/ SOL(-) cases, we identified 2 pattern-associated proteins that were consistent with previous findings of adhesion molecules disassembling, as well as apoptosis resistance engagement in the aggressive and metastatic behavior of MIP/SOL growth ADC. ${ }^{14-16}$ The presence of either of these proteins, IGFBP-2 and P-cadherin, to define MIP/ SOL positivity in the SDB test also showed superiority than the presence of both of them to indicate a positive result (Table E4).

Nodal upstaging was found in $22.1 \%$ of the patients in the current study, which is higher than $18.6 \%$ in a previous study. ${ }^{23}$ Two reasons could explain the higher nodal upstaging rate in this article. On the one hand, the average number of lymph nodes resected in our series was more than 10 , thus increasing the chance of detecting occult nodal metastasis. On the other hand, approximately half of the patients had MIP or SOL growth patterns, and these tumors had more aggressive biological behavior to have lymph node metastasis despite the limited tumor size. ${ }^{6}$ Of note, $45.4 \%$ of the tumors with false-negative results had N1 station metastasis, highlighting the importance of evaluating hilar region intraoperatively if the SDB test shows a negative result.

\section{Study Limitations}

The diagnostic kit proposed in the current study can give a rapid detection of MIP/SOL pattern by chromogen density comparison, providing an adjuvant tool to guide individualized resection strategy for early-stage lung ADCs (Figure 4). However, several limitations of this study should be addressed. First, the mean tumor size was greater, which is the general indication for sublobar resection of $2 \mathrm{~cm}$, although we recruited clinical stage I cases with tumors no more than $2 \mathrm{~cm}$ in the prospective cohort. The larger diameters than their preoperative appearances may be partly explained by the time interval ( $\sim 2$ months in some cases) between CT scan and surgery in our public hospitals. Second, the subjective interpretation of the SDB result, done by comparing chromogen density, rendered interobserver bias unavoidable. One potential solution in future work for minimizing this interobserver disagreement is to use a densitometer and conduct sensitivity testing of various densitometry thresholds to determine the appropriate level to be used, which might semi-quantify the results of defining positive results. The 1-step nucleic acid amplification system, which semiquantitatively assesses mRNA markers, could be a useful alternative and objective method, although its cost is 10 -fold higher than the approximate $\$ 15$ per case needed in the SDB test. ${ }^{21,24}$ Third, similar to frozen sections, sampling error was inevitable, because only part of each removed tumor was examined. A more consistent tumor sampling method, for example, systematically take section every $3 \mathrm{~mm}$ along the tumor, may help increase the sensitivity and reduce sampling error. We were unable to determine whether false-negative results in the present study were due to nonexpression of the marker proteins or to sampling error, that is, tissue sampled did not include the MIP/SOL region within the lesion. The combination of both frozen section and SDB methods may improve the accuracy of detection in the future. Last, we found only 2 markers from screening 61 proteins of 2 molecular pathways in this study. It is theoretically possible that if more MIP/SOL-specific proteins are discovered in the future, the sensitivity of the diagnostic kit could be improved accordingly.

\section{CONCLUSIONS}

A novel diagnostic kit developed on the basis of the SDB method was established in the present study. The test, when used to identify MIP/SOL components in a prospective cohort consisting of 68 ADCs, achieved high specificity $(94.4 \%)$ and moderate sensitivity $(65.6 \%)$. The test can be performed in less than 30 minutes and could be used as an alternative method for detecting MIP/SOL intraoperatively. These initial results should be validated in further larger multicenter cohorts.

\section{Conflict of Interest Statement}

Authors have nothing to disclose with regard to commercial support.

The authors thank the staff members of the surgical laboratory in the Prince of Wales Hospital, Hong Kong, for their assistance in preparing cancer specimens.

\section{References}

1. Zhao ZR, Situ DR, Lau RWH, Mok TSK, Chen GG, Underwood MJ, et al. Comparison of segmentectomy and lobectomy in stage IA adenocarcinomas. J Thorac Oncol. 2017;12:890-6.

2. Nakamura K, Saji H, Nakajima R, Okada M, Asamura H, Shibata T, et al. A phase III randomized trial of lobectomy versus limited resection for small-sized peripheral non-small cell lung cancer (JCOG0802/WJOG4607L). Jpn J Clin Oncol. 2010;40:271-4.

3. Ng CS, Zhao ZR, Lau RW. Tailored therapy for stage I non-small-cell lung cancer. J Clin Oncol. 2017;35:268-70.

4. Russell PA, Wainer Z, Wright GM, Daniels M, Conron M, Williams RA. Does lung adenocarcinoma subtype predict patient survival?: a clinicopathologic study based on the new International Association for the Study of Lung Cancer/American Thoracic Society/European Respiratory Society international multidisciplinary lung adenocarcinoma classification. J Thorac Oncol. 2011;6:1496-504.

5. Zhao ZR, Xi SY, Li W, Situ DR, Chen KM, Yang H, et al. Prognostic impact of pattern-based grading system by the new IASLC/ATS/ERS classification in Asian patients with stage I lung adenocarcinoma. Lung Cancer. 2015;90:604-9. 
6. Zhao ZR, To KF, Mok TS, Ng CS. Is there significance in identification of nonpredominant micropapillary or solid components in early-stage lung adenocarcinoma? Interact Cardiovasc Thorac Surg. 2017;24:121-5.

7. Huang KY, Ko PZ, Yao CW, Hsu CN, Fang HY, Tu CY, et al. Inaccuracy of lung adenocarcinoma subtyping using preoperative biopsy specimens. J Thorac Cardiovasc Surg. 2017;154:332-9.

8. Cha MJ, Lee HY, Lee KS, Jeong JY, Han J, Shim YM, et al. Micropapillary and solid subtypes of invasive lung adenocarcinoma: clinical predictors of histopathology and outcome. J Thorac Cardiovasc Surg. 2014;147:921-8.

9. Yeh YC, Nitadori J, Kadota K, Yoshizawa A, Rekhtman N, Moreira AL, et al. Using frozen section to identify histological patterns in stage I lung adenocarcinoma of $</=3 \mathrm{~cm}$ : accuracy and interobserver agreement. Histopathology. 2015; 66:922-38.

10. Trejo Bittar HE, Incharoen P, Althouse AD, Dacic S. Accuracy of the IASLC/ ATS/ERS histological subtyping of stage I lung adenocarcinoma on intraoperative frozen sections. Mod Pathol. 2015;28:1058-63.

11. Travis WD, Brambilla E, Noguchi M, Nicholson AG, Geisinger KR, Yatabe Y, et al. International Association for the Study of Lung Cancer/American Thoracic Society/European Respiratory Society international multidisciplinary classification of lung adenocarcinoma. J Thorac Oncol. 2011;6:244-85.

12. Travis WD, Brambilla E, Nicholson AG, Yatabe Y, Austin JHM, Beasley MB, et al. The 2015 World Health Organization classification of lung tumors: impact of genetic, clinical and radiologic advances since the 2004 classification. $J$ Thorac Oncol. 2015;10:1243-60.

13. Travis WD, Brambilla E, Rami-Porta R, Vallières E, Tsuboi M, Rusch V, et al. Visceral pleural invasion: pathologic criteria and use of elastic stains: proposal for the 7th edition of the TNM classification for lung cancer. J Thorac Oncol. 2008;3:1384-90.

14. Kamiya K, Hayashi Y, Douguchi J, Hashiguchi A, Yamada T, Izumi Y, et al. Histopathological features and prognostic significance of the micropapillary pattern in lung adenocarcinoma. Mod Pathol. 2008;21:992-1001.

15. Nagano T, Ishii G, Nagai K, Ito T, Kawase A, Takahashi K, et al. Structural and biological properties of a papillary component generating a micropapillary component in lung adenocarcinoma. Lung Cancer. 2010;67:282-9.
16. Sun X, Wei P, Shen C, Yang Y, Wang Y, Li Y, et al. Prognostic value of the IASLC/ATS/ERS classification and IMP3 expression in lung adenocarcinoma of Chinese cases. Am J Cancer Res. 2015;5:2266-76.

17. Hirakawa H, Shibata K, Ohzono E. Use of a semi-dry dot-blot for rapid detection of lymph node metastasis. Clin Chim Acta. 2010;411:1149-50.

18. Warth A, Muley T, Meister M, Stenzinger A, Thomas M, Schirmacher P, et al. The novel histologic International Association for the Study of Lung Cancer/ American Thoracic Society/European Respiratory Society classification system of lung adenocarcinoma is a stage-independent predictor of survival. J Clin Oncol. 2012;30:1438-46.

19. Nitadori J, Bograd AJ, Kadota K, Sima CS, Rizk NP, Morales EA, et al. Impact of micropapillary histologic subtype in selecting limited resection vs lobectomy for lung adenocarcinoma of $2 \mathrm{~cm}$ or smaller. J Natl Cancer Inst. 2013;105:1212-20.

20. Aliyev N, Takahashi Y, Lu S, Mayor M, Adusumilli PS. Intraoperative subtyping of lung adenocarcinoma: an unmet need. Transl Cancer Res. 2016;S40-3.

21. Tomoshige K, Tsuchiya T, Otsubo R, Oikawa M, Yamasaki N, Matsumoto K, et al. Intraoperative diagnosis of lymph node metastasis in non-small-cell lung cancer by a semi-dry dot-blot method. Eur J Cardiothorac Surg. 2016;49:617-22.

22. Otsubo R, Oikawa M, Hirakawa H, Shibata K, Abe K, Hayashi T, et al. Novel diagnostic procedure for determining metastasis to sentinel lymph nodes in breast cancer using a semi-dry dot-blot method. Int J Cancer. 2014;134:905-12.

23. Licht PB, Jørgensen OD, Ladegaard L, Jakobsen E. A national study of nodal upstaging after thoracoscopic versus open lobectomy for clinical stage I lung cancer. Ann Thorac Surg. 2013:96:943-50.

24. Inoue M, Hiyama K, Nakabayashi K, Morii E, Minami M, Sawabata N, et al. An accurate and rapid detection of lymph node metastasis in non-small cell lung cancer patients based on one-step nucleic acid amplification assay. Lung Cancer. 2012; $78: 212-8$.

Key Words: early-stage lung cancer, ground-glass opacity, lung adenocarcinoma, micropapillary, semi-dry dot-blot, solid 
TABLE E1. Array maps showing the proteins screened in adhesion pathway

\begin{tabular}{|c|c|c|c|c|c|c|c|c|c|c|}
\hline & $\mathbf{A}$ & B & C & D & $\mathbf{E}$ & $\mathbf{F}$ & G & $\mathbf{H}$ & I & $\mathbf{J}$ \\
\hline 1 & Pos1 & BCAM & E-cadherin & E-selectin & ICAM-2 & L-selectin & NrCAM & PECAM-1 & VCAM-1 & $\mathrm{Neg}$ \\
\hline \multicolumn{11}{|c|}{2} \\
\hline \multicolumn{11}{|c|}{3} \\
\hline \multicolumn{11}{|l|}{4} \\
\hline 5 & Pos 2 & ALCAM & CEACAM-1 & TROP1 & ICAM-1 & ICAM-3 & NCAM-1 & P-cadherin & P-selectin & Cadherin \\
\hline \multicolumn{11}{|l|}{6} \\
\hline \multicolumn{11}{|l|}{7} \\
\hline 8 & & & & & & & & & & \\
\hline
\end{tabular}

Pos, Positive control; Neg, negative control.

TABLE E2. Array maps showing the proteins screened in apoptosis pathway

\begin{tabular}{|c|c|c|c|c|c|c|c|c|c|c|c|c|c|}
\hline & $\mathbf{A}$ & B & C & D & $\mathbf{E}$ & $\mathbf{F}$ & G & $\mathbf{H}$ & I & $\mathbf{J}$ & $\mathbf{K}$ & $\mathbf{L}$ & $\mathbf{M}$ \\
\hline 1 & Pos1 & Pos2 & Pos 3 & $\mathrm{Neg}$ & $\mathrm{Neg}$ & bad & bax & bcl-2 & bcl-w & BID & BIM & Caspase3 & Caspase8 \\
\hline \multicolumn{14}{|l|}{2} \\
\hline 3 & $\mathrm{CD} 40$ & $\mathrm{CD} 40 \mathrm{~L}$ & cIAP-2 & cytoC & DR6 & Fas & FasL & neg & HSP27 & HSP60 & HSP70 & HTRA & IGF-1 \\
\hline \multicolumn{14}{|c|}{4} \\
\hline 5 & IGF-II & IGFBP-1 & IGFBP-2 & IGFBP-3 & IGFBP-4 & IGFBP-5 & IGFBP-6 & IGF-1sR & livin & p21 & p27 & $\mathrm{p} 53$ & SMAC \\
\hline \multicolumn{14}{|l|}{6} \\
\hline 7 & Survivin & sTNF-R1 & sTNF-R2 & TNF-alpha & TNF-beta & TRAILR-1 & TRAILR-2 & TRAILR-3 & TRAILR-4 & XIAP & Neg & Neg & Neg \\
\hline 8 & & & & & & & & & & & & & \\
\hline
\end{tabular}

TABLE E3. Antibody array results for micropapillary/solid positive tumor against negative tumor

\begin{tabular}{|c|c|c|c|c|c|c|}
\hline \multirow[b]{2}{*}{ Protein } & \multicolumn{2}{|c|}{ Micropapillary } & \multicolumn{2}{|c|}{ Solid } & \multicolumn{2}{|c|}{ Micropapillary + solid } \\
\hline & Fold-change & $P$ value & Fold-change & $P$ value & Fold-change & $P$ value \\
\hline IGFBP2* & 2.81 & .03 & 3.46 & $<.01$ & 3.14 & $<.01$ \\
\hline P-cadherin* & 2.35 & .01 & 2.58 & .05 & 2.46 & .03 \\
\hline sTNF-R1 & 1.90 & .01 & 0.90 & .85 & 1.62 & .05 \\
\hline HSP27 & 2.42 & .01 & 0.30 & .18 & 1.86 & .02 \\
\hline
\end{tabular}

IGFBP2, Insulin-like growth factor-binding protein 2; $s T N F-R 1$, soluble tumor necrosis factor receptor 1; HSP, heat shock protein. *Validated by Western blot.

TABLE E4. Comparison of different definition of positive result in semi-dry dot-blot test

\begin{tabular}{|c|c|c|c|c|c|c|}
\hline \multirow[b]{2}{*}{ Definition of positivity } & \multirow[b]{2}{*}{ Sensitivity $\%(95 \%$ CI $)$} & \multirow[b]{2}{*}{ Specificity $\%(95 \%$ CI $)$} & & \multicolumn{2}{|c|}{ Pathologic assessment } & \multirow[b]{2}{*}{ Sum } \\
\hline & & & & MIP/SOL positive & MIP/SOL negative & \\
\hline \multirow[t]{2}{*}{ Either P-cadherin or IGFBP2 } & $65.6(46.8-81.4)$ & $94.4(81.3-99.3)$ & Positive & 21 & 2 & 23 \\
\hline & & & Negative & 11 & 34 & 45 \\
\hline \multirow[t]{2}{*}{ Both P-cadherin and IGFBP2 } & $34.4(18.6-53.2)$ & $97.2(85.5-99.9)$ & Positive & 11 & 1 & 12 \\
\hline & & & Negative & 21 & 35 & 56 \\
\hline \multirow[t]{2}{*}{ Only P-cadherin } & $43.8(26.4-62.3)$ & $97.2(85.5-99.9)$ & Positive & 14 & 1 & 15 \\
\hline & & & Negative & 18 & 35 & 53 \\
\hline \multirow[t]{2}{*}{ Only IGFBP2 } & $56.3(37.7-73.6)$ & $94.4(81.3-99.3)$ & Positive & 18 & 2 & 20 \\
\hline & & & Negative & 14 & 34 & 48 \\
\hline Sum & & & & 32 & 36 & 68 \\
\hline
\end{tabular}

CI, Confidence interval; $M I P$, micropapillary; SOL, solid; IGFBP2, insulin-like growth factor-binding protein 2. 
TABLE E5. Semi-dry dot-blot test for identifying micropaillary or solid pattern separately

\begin{tabular}{|c|c|c|c|c|c|c|}
\hline \multirow[b]{2}{*}{ SDB test } & \multirow[b]{2}{*}{ Sensitivity \% $(95 \%$ CI $)$} & \multirow[b]{2}{*}{ Specificity \% (95\% CI) } & & \multicolumn{2}{|c|}{ Pathologic assessment } & \multirow[b]{2}{*}{ Sum } \\
\hline & & & & Positive & Negative & \\
\hline \multirow[t]{3}{*}{ MIP } & $69.2(38.6-90.9)$ & $74.6(61.0-85.3)$ & Positive & 9 & 14 & 23 \\
\hline & & & Negative & 4 & 41 & 45 \\
\hline & & & Sum & 13 & 55 & 68 \\
\hline \multirow[t]{3}{*}{ SOL } & $60.0(36.1-80.9)$ & $77.1(62.7-88.0)$ & Positive & 12 & 11 & 23 \\
\hline & & & Negative & 8 & 37 & 45 \\
\hline & & & Sum & 20 & 48 & 68 \\
\hline
\end{tabular}

Note that 1 case had both MIP and SOL component. SDB, Semi-dry dot-blot test; $C I$, confidence interval; MIP, micropapillary; SOL, solid. 